\title{
Study of the Modulating Interactions of Multitrait Rhizobacteria Using Zea Mays L. as the Host Plant
}

\author{
Sana Shakeel, Ambreen Ahmed*, Ifrah Javaid \\ Department of Botany, University of the Punjab, Quaid-e-Azam Campus, Lahore 54590, Pakistan
}

\section{ABSTRACT}

Background: Rhizosphere is a soil region closest to roots of the plants inhabiting different types of microorganisms including rhizobacteria. Chemical fertilizers which are conventionally used for increasing crop production are dangerous in terms of minimizing the nutritional value of crops and may also be hazardous for biological agents. Therefore, the use of Plant Growth Promoting Rhizobacteria (PGPR) are favorable for improved crop production over chemical fertilizers.

Objectives: The current study highlights the growth promoting traits of bacterial isolates through isolation of rhizospheric bacterial strains from different plants.

Methodology: In this study, ten rhizospheric bacterial isolates were used, which were morpho-physiologically characterized and then tested for plant growth-promoting traits i.e., $\mathrm{HCN}$ production, ammonification and auxin production. Most of the bacterial strains gave positive results for these plant growth-promoting traits. To study the beneficial effects of these bacteria on plants, plantmicrobial interaction assay was conducted using Zea mays.

Results: Results revealed that these bacteria enhanced the growth as compared to control plants. Bacterial isolates Streptomyces lydicus (Cn6), Staphylococcus aureus (Cn7) and Bacillus pumilus (PP3) showed strong ammonia producing effects. The isolates Bacillus subtilis (Cn2), PP2 and PP5 exhibited strong potential of HCN production whereas only Streptomyces lydicus (Cn6) and Bacillus pumilus (PP3) were observed to be auxin producers. A maximum increase in fresh weight of the plants was observed in treatment with PP2 showing $94.36 \%$ increase over controls. Cn1 showed an increase $(26.12 \%)$ in shoot length while $\mathrm{Cn} 5$ revealed a prominent increase (64.95\%) in root length compared to the control plant. The isolates $\mathrm{Cn} 5$ and $\mathrm{Cn} 4$ showed improvement in the total chlorophyll content of the treated plants with a percentage increase of $100 \%$ and $99.82 \%$, respectively compared to the control. Conclusion: In conclusion, these PGPR may be further used in agriculture research for growth improvement.

Keywords *Address of Correspondence Article info.

Rhizobacteria, PGP, plant-microbial interaction assay, PGPR, Zea mays.

Cite this article: Shakeel S, Ahmed A, Javaid I. Study of the Modulating Interactions of Multitrait Rhizobacteria Using Zea Mays L. as the Host Plant. RADS J Biol Res Appl Sci. 2019; 10(2):44-53.

This is an Open Access article distributed under the terms of the Creative Commons Attribution License (http://creativecommons.org/licenses/by/4.0), which permits unrestricted use, distribution, and reproduction in any medium, provided the original work is properly cited.

\section{INTRODUCTION}

The relationship between species is frequently defined via the influence of associations and interactions among species. Plants live in an association with a rich variety of microorganisms throughout their development. These associations relate to plant-microbe interactions from mutualism to parasitism, on account of the role of symbionts ${ }^{1}$. Different groups of bacteria and fungi interact with higher plants. While studying plants and microbes, rhizosphere is a critical segment of soil. In plants, the rhizosphere is a multifarious and diverse environment occupied by numerous microbes ${ }^{2}$. The root colonizing bacteria are known as rhizospheric bacteria ${ }^{3}$.

Different species of rhizobacteria that flourish in the rhizosphere are able to stimulate plant growth by different 
mechanisms and are collectively known as plant growthpromoting rhizobacteria/rhizospheric bacteria (PGPR) ${ }^{4}$. In the past few years, the number of PGPR that has been recognized has shown a significant increase, largely because the part of rhizosphere in an ecosystem has attained prominence in the working of the biosphere. Many species of bacteria like Azospirillum, Azotobacter, Pseudomonas, Enterobacter, Serratia, Klebsiella, Burkholderia, Alcaligenes, Arthrobacter and Bacillus species have been testified to increase plant growth ${ }^{5}$. Whenever the roots are inoculated with a PGPR, reduced growth rate of the primary roots and increased number and/or length of lateral roots may be observed 6 . Accordingly, through improved uptake of water and minerals, the growth of the whole plant can be augmented. A number of these effects, including augmented root and shoot biomass, are also acknowledged for PGPR-inoculated plants growing in soil 7,8 . PGPR amend RSA and the assembly of roots, chiefly via their capability to meddle with the plant hormonal balance? .

PGPR utilization meets the current need to lessen nitrogen input to achieve better sustainability in the production of crops, predominantly cereals ${ }^{10}$. Maize (Zea mays L.) is one of the major crops all over the globe reserving the mounting claim for food and cultivated animals feed ${ }^{11}$. Exhaustive corn production requires broad consumption of pesticides as well as chemical fertilizers, which have harmful effects on the soil, water and on the food chain. It has been verified that although chemical fertilizers improve the crop yield, they cause toxic effects too, for instance soil acidification as well as production of the greenhouse gas, nitrous oxide $\left(\mathrm{N}_{2} \mathrm{O}\right)$ by denitrification on ecosystems ${ }^{12,13}$. It has been reported that one possible mode to reduce the harmful environmental impacts ensuing from continuous utilization of chemical fertilizers is inoculation with biofertilizers such as PGPR ${ }^{14}$. Several findings have revealed that PGPR are latent growth enhancers in numerous crops ${ }^{15}$.

The present study deals with four already isolated and identified bacterial strains [Bacillus subtilis (Cn2), Streptomyces lydicus (Cn6), Staphylococcus aureus (Cn7) and Bacillus pumilus (PP3)] and isolation of six rhizospheric bacterial strains from plants Convolvulus arvensis and Polygonum plebium collected from
Balochistan, highlighting the growth promoting traits of bacterial isolates. These bacterial isolates were then used to inoculate the seeds of Zea mays and various parameters of treated and non-treated plants were studied regarding plant growth improvement.

\section{MATERIALS AND METHODS}

\section{Sample Collection}

For the isolation of bacteria, Convolvulus arvensis and Polygonum plebeium plants were collected from the Zhob district of Balochistan (Table 1).

\section{Isolation and Characterization of Bacteria}

Six bacterial strains were isolated from the rhizosphere of the collected plant using the serial dilution method (Table 1). The isolates were routinely grown on L-Agar (Luria Agar) or L-Broth (Luria Bertani Broth). The cultures were purified by repeated streaking on L-Agar medium.

All the bacterial strains including four already isolated and identified strains [Bacillus subtilis (Cn2), Streptomyces lydicus (Cn6), Staphylococcus aureus (Cn7) and Bacillus pumilus (PP3)] were characterized morphologically and physiologically. Culture characteristics as well as cell morphology was observed. To examine bacterial physiology, growth curve, temperature and $\mathrm{pH}$, the studies were conducted following the research of Fatima and Ahmed $^{16}$.

\section{Plant Growth-Promoting Traits}

To determinate plant growth-promoting traits, tests for ammonification, HCN production and auxin production were performed following the research of Cappuccino and Sherman ${ }^{17}$; Lorck ${ }^{18}$ and Ahmed and Hasnain ${ }^{19}$.

\section{Plant-Microbe Interactions}

Ten strains were used in the plant-microbe interaction experiment and their effect on plant growth was observed by recording various growth parameters following the study by Fatima and Ahmed ${ }^{16}$. The experiment was repeated thrice.

\section{Statistical Analysis}

The data obtained were statistically analyzed using the software SPSS v. 16. 


\section{RESULTS}

\section{Isolation and Characterization of Bacteria}

For rhizospheric soil samples, Convolvulus arvensis and Polygonum plebeium plants were collected from the Zhob district of Balochistan. Six bacterial strains were isolated from the rhizosphere of the selected plants through the serial dilution method and were used for further study including four already isolated strains [Bacillus subtilis (Cn2), Streptomyces lydicus (Cn6), Staphylococcus aureus $(\mathrm{Cn} 7)$ and Bacillus pumilus (PP3)]. Different bacterial isolates showed different colony size, shape, color, texture, elevation, margin and growth. Bacterial isolates $\mathrm{Cn} 1$, Bacillus subtilis (Cn2), Cn4, Staphylococcus aureus (Cn7) and PP5 showed irregular shape; Cn5, Streptomyces lydicus (Cn6), PP2 and Bacillus pumilus (PP3) were round in shape, while only PP4 possessed filamentous shape. All the bacterial isolates were gram +ve and except for Cn1, Bacillus pumilus (PP3), PP4 and PP5, all were cocci (Table 1).

For physiological characterization, growth curve, temperature test and $\mathrm{pH}$ studies were performed. The growth curve revealed that all the strains gave optimal growth after 24 and 48 hours of incubation whereas a significant decline was observed after 72 and 96 hours of incubation. Bacterial strains Bacillus subtilis (Cn2), Cn5, Staphylococcus aureus (Cn7) and PP5 showed maximum growth after 24 hours of incubation while maximum growth of strains Cn1, Streptomyces lydicus (Cn6), Cn4, PP2, Bacillus pumilus (PP3) and PP4 was observed after 48 hours of incubation (Figure 1). The temperature test showed that all the strains gave optimal growth at $37^{\circ} \mathrm{C}$ temperature while bacterial growth was observed to decrease at $25^{\circ} \mathrm{C}$ and $45^{\circ} \mathrm{C}$, respectively (Figure 2). All the strains showed different behavior towards different $\mathrm{pH}$ ranges from 5, 6, 7, 8 and 9. Optimum $\mathrm{pH}$ for growth of bacterial isolate PP2 was pH5. On the other hand, the bacterial strains $\mathrm{Cn} 1$, Bacillus subtilis (Cn2), Cn5 and Streptomyces lydicus ( $\mathrm{Cn} 6)$ showed maximum growth at $\mathrm{pH} 7$, Staphylococcus aureus $(\mathrm{Cn} 7)$ at $\mathrm{pH} 8$, while $\mathrm{Cn} 4$ and PP5 showed maximum growth at pH9 (Figure 3 ).

\section{Plant Growth-Promoting Traits}

All the bacterial isolates showed positive ammonification test except for Cn5 and PP4. Only Streptomyces lydicus $(\mathrm{Cn} 6)$ bacterial strain gave negative results for $\mathrm{HCN}$ production whereas all other strains were $\mathrm{HCN}$ producers. On the other hand, only bacterial strains Streptomyces lydicus (Cn6) and Bacillus pumilus (PP3) were auxin producers while rest of the strains showed negative results for auxin production (Table 1).

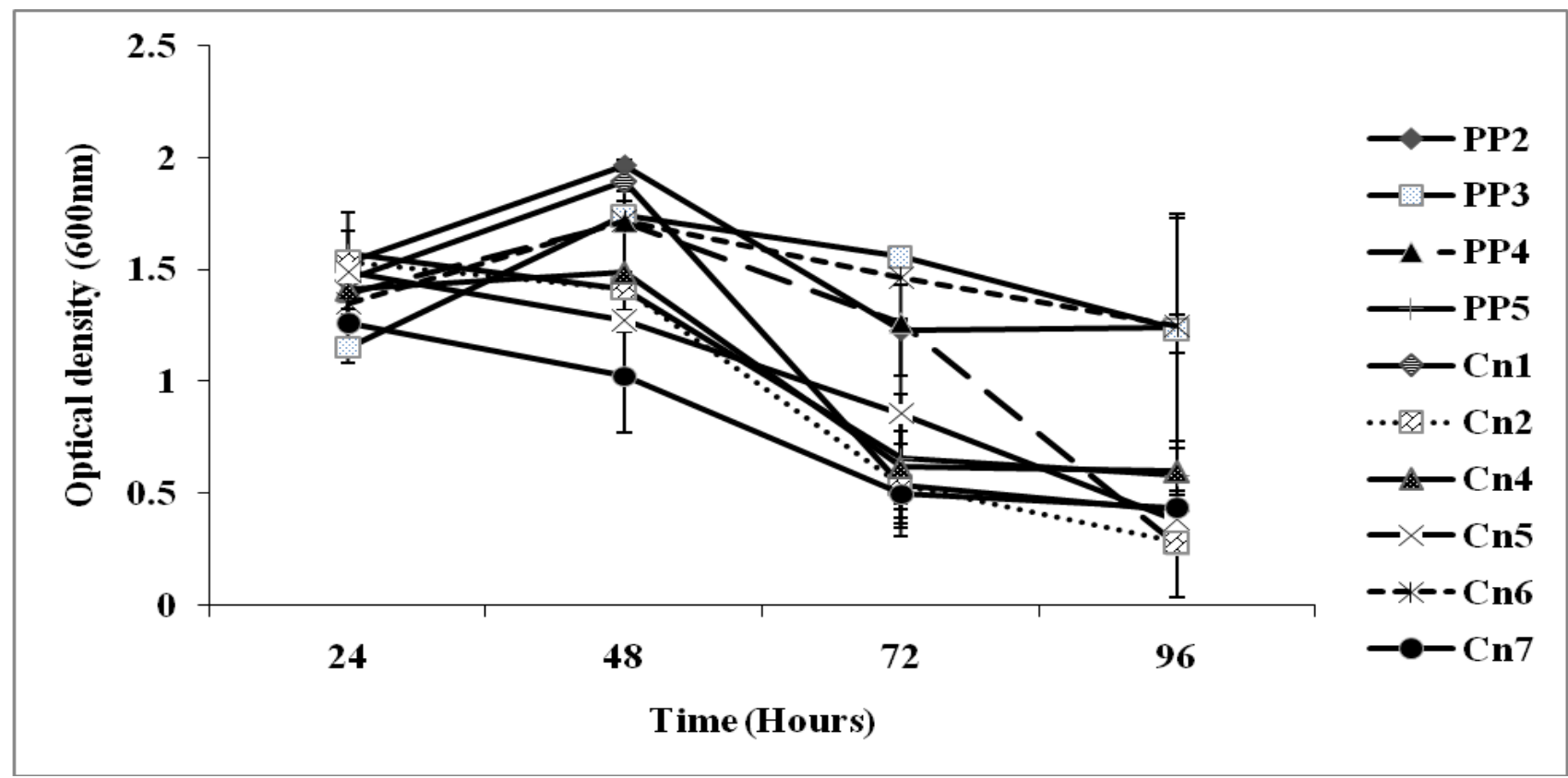

Figure 1. Growth curve of bacterial strains in L-Broth medium for different intervals of time (24, 48, 72 and 96 hours). 


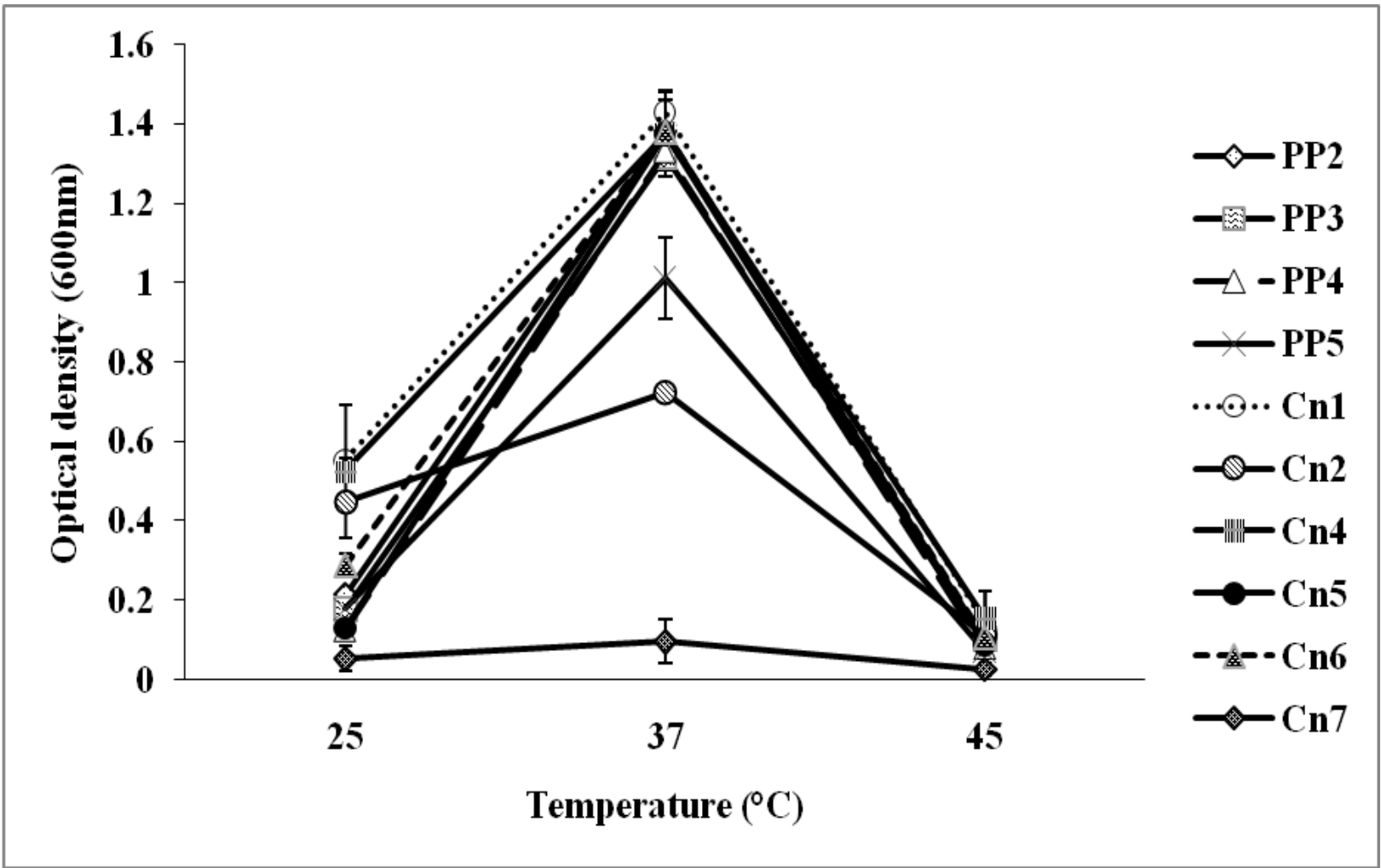

Figure 2. Growth of bacterial strains grown in L-Broth medium at different temperatures $\left(25,37\right.$ and $\left.45^{\circ} \mathrm{C}\right)$.

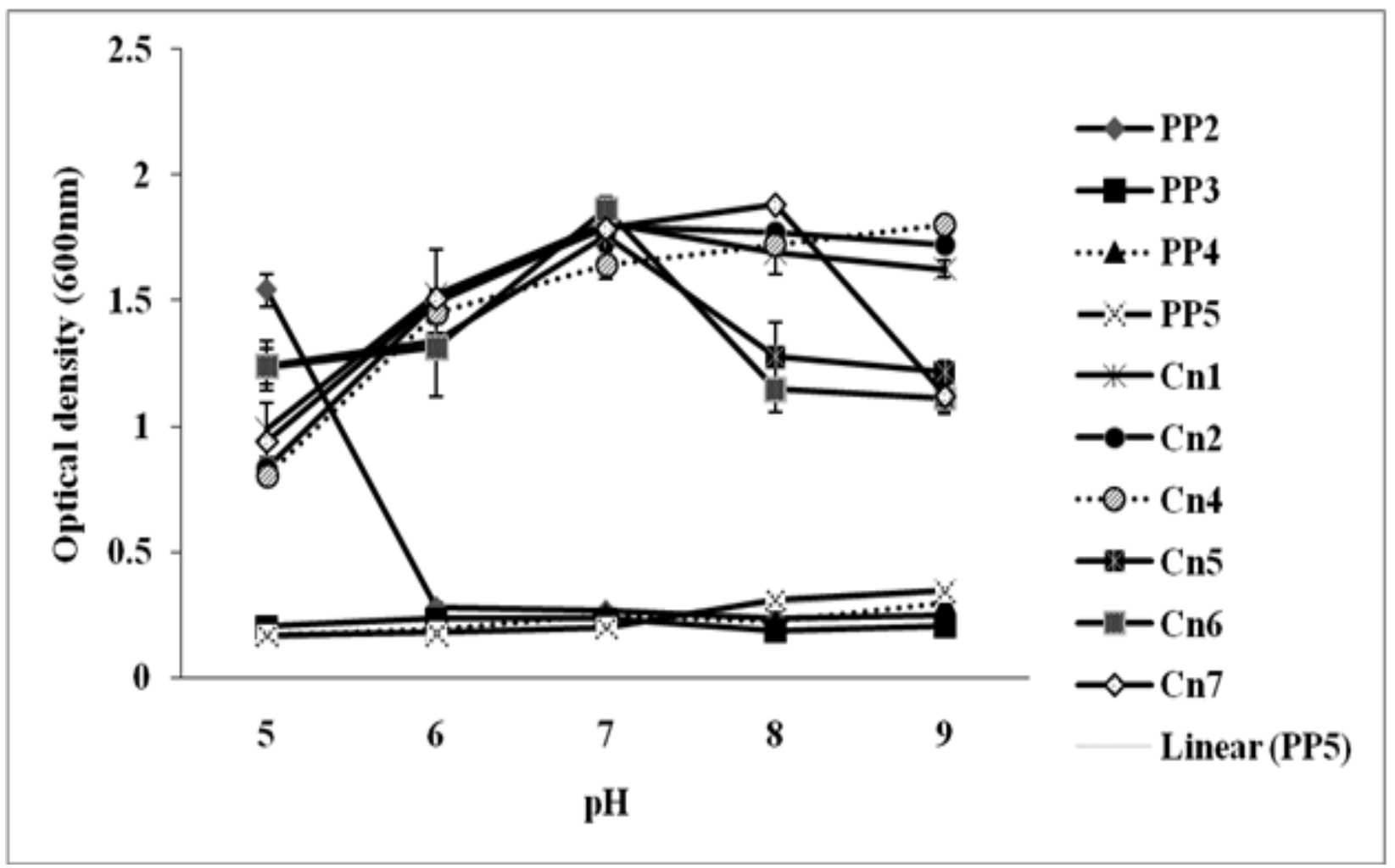

Figure 3. Growth of bacterial strains in L-Broth medium with $\mathrm{pH}$ range $(5,6,7,8$ and 9$)$. 
Table 1. Morphological characterization of bacterial isolates.

\begin{tabular}{|c|c|c|c|c|c|c|c|c|c|c|c|}
\hline & 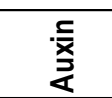 & ' & & ' & ' & 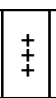 & ' & ' & $\stackrel{+}{+}$ & ' & ' \\
\hline \multirow{2}{*}{$\begin{array}{l}0 \\
0 \\
\frac{\pi}{3} \\
0 \\
0 \\
0 \\
0\end{array}$} & 어 & + & $\stackrel{\ddagger}{\ddagger}$ & + & + & ' & + & $\stackrel{\ddagger}{\ddagger}$ & + & + & $\stackrel{+}{+}$ \\
\hline & 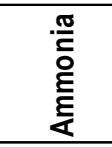 & $\ddagger$ & $\ddagger$ & $\ddagger$ & ' & 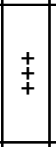 & $\stackrel{\ddagger}{+}$ & + & $\stackrel{\ddagger}{+}$ & ' & $\ddagger$ \\
\hline \multirow{3}{*}{ 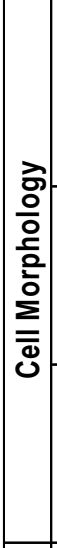 } & 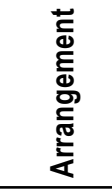 & 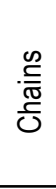 & 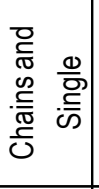 & 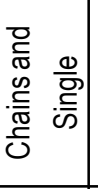 & 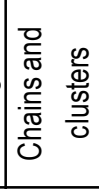 & 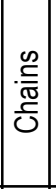 & 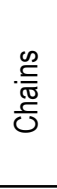 & \begin{tabular}{|l}
$\frac{0}{0}$ \\
$\frac{5}{5}$ \\
के
\end{tabular} & 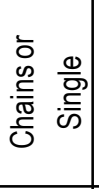 & 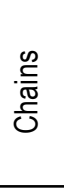 & $\begin{array}{l}\frac{0}{\pi} \\
\frac{\pi}{0} \\
\frac{\pi}{0}\end{array}$ \\
\hline & 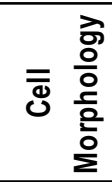 & \begin{tabular}{l} 
D \\
D \\
\hdashline
\end{tabular} & $\begin{array}{l}\bar{U} \\
\bar{O}\end{array}$ & $\begin{array}{l}\bar{U} \\
\bar{O}\end{array}$ & $\begin{array}{l}\bar{U} \\
\bar{\delta}\end{array}$ & \begin{tabular}{|l}
$\bar{J}$ \\
$\bar{u}$ \\
$\mathcal{u}$
\end{tabular} & $\begin{array}{l}\bar{J} \\
\text { J }\end{array}$ & \begin{tabular}{|l|}
$\overline{0}$ \\
$\bar{\delta}$ \\
$\mathcal{O}$
\end{tabular} & $\begin{array}{l}\text { D } \\
\text { O } \\
\square\end{array}$ & 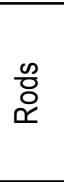 & 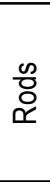 \\
\hline & 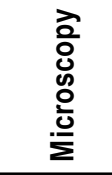 & $\begin{array}{l}\stackrel{0}{+} \\
+ \\
\stackrel{+}{E} \\
\frac{\pi}{0} \\
0\end{array}$ & 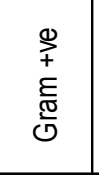 & 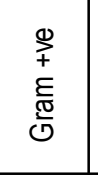 & 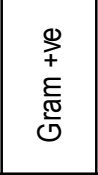 & 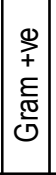 & 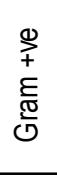 & 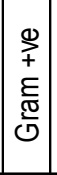 & 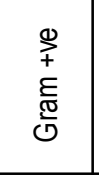 & 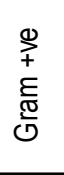 & 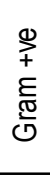 \\
\hline \multirow{10}{*}{ 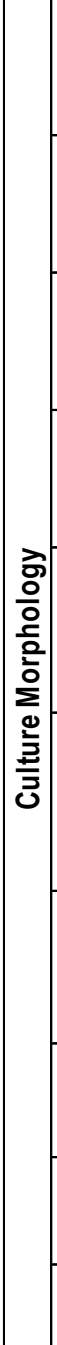 } & $\begin{array}{l}\text { 売 } \\
\text { ơ }\end{array}$ & $\begin{array}{l}\frac{0}{\pi} \\
\frac{\pi}{0} \\
\frac{0}{2} \\
\Sigma\end{array}$ & $\begin{array}{l}\frac{0}{\frac{\pi}{\pi}} \\
\frac{\pi}{0} \\
\frac{0}{2} \\
\sum\end{array}$ & $\begin{array}{l}\frac{\Phi}{\pi} \\
\frac{\tilde{0}}{0} \\
\frac{0}{0} \\
\sum\end{array}$ & $\begin{array}{l}\frac{\Phi}{\pi} \\
\frac{\tilde{0}}{0} \\
\frac{0}{0} \\
\sum\end{array}$ & $\frac{\overline{0}}{\overline{\bar{z}}}$ & $\frac{\frac{0}{0}}{\frac{0}{0}}$ & \begin{tabular}{|l|}
$\frac{0}{\pi}$ \\
$\frac{\pi}{0}$ \\
$\frac{0}{0}$ \\
$\frac{0}{2}$ \\
\end{tabular} & $\frac{\bar{d}}{\frac{0}{0}}$ & $\begin{array}{l}\frac{\bar{d}}{\frac{d}{0}} \\
\frac{0}{20}\end{array}$ & $\frac{\overline{0}}{\overline{\bar{z}}}$ \\
\hline & 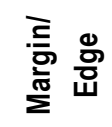 & 总 & 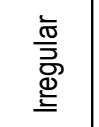 & 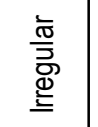 & 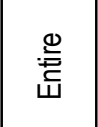 & $\begin{array}{l}\text { 黑 } \\
\text { 立 }\end{array}$ & 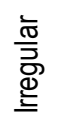 & 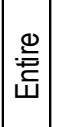 & 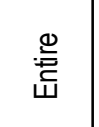 & 莺 & 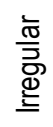 \\
\hline & 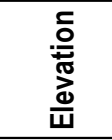 & 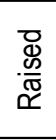 & 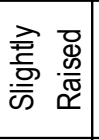 & $\frac{\pi}{\frac{\pi}{L}}$ & 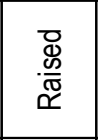 & 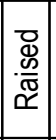 & $\frac{\pi}{\frac{\pi}{L}}$ & 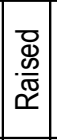 & 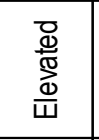 & $\frac{\pi}{\frac{\pi}{4}}$ & $\frac{\pi}{\frac{\pi}{4}}$ \\
\hline & 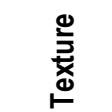 & $\begin{array}{l}\text { Fे } \\
\text { है } \\
\text { के }\end{array}$ & $\begin{array}{l}\text { Fै } \\
\text { ठ } \\
\text { के }\end{array}$ & $\begin{array}{l}\text { Fే } \\
\text { ष्ठ } \\
\text { क }\end{array}$ & $\begin{array}{l}\text { F्ञ } \\
\text { ठ } \\
\text { क }\end{array}$ & 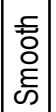 & $\begin{array}{l}\bar{\Xi} \\
\frac{\Phi}{\grave{2}} \\
\text { के }\end{array}$ & $\begin{array}{l}\overline{7} \\
\bar{d} \\
\stackrel{0}{0} \\
\text { की }\end{array}$ & $\begin{array}{l}\text { Fే } \\
\text { ठ } \\
\text { के }\end{array}$ & $\begin{array}{l}\text { 表 } \\
\sum\end{array}$ & 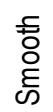 \\
\hline & 흥 & 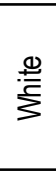 & 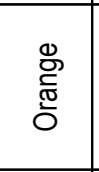 & 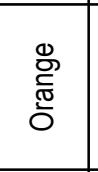 & 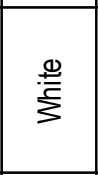 & 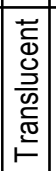 & 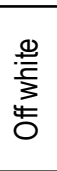 & 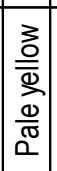 & 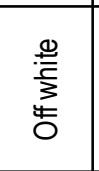 & 产 & $\begin{array}{l}\mathscr{8} \\
\stackrel{\bar{\pi}}{\pi} \\
\overline{0}\end{array}$ \\
\hline & $\begin{array}{l}\frac{\Xi}{\pi} \\
\frac{\delta}{\infty}\end{array}$ & 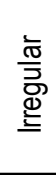 & 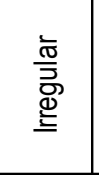 & 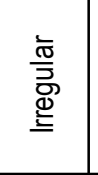 & 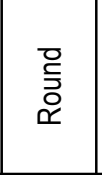 & 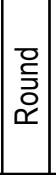 & 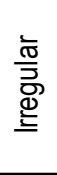 & 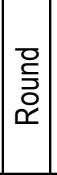 & $\begin{array}{l}\text { 을 } \\
\text { ড় } \\
\text { ه }\end{array}$ & 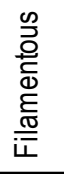 & 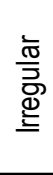 \\
\hline & 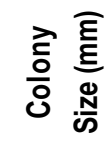 & - & 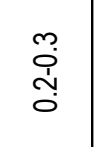 & 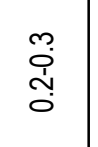 & $\stackrel{1}{\circ}$ & $\mid \begin{array}{c}0 \\
0 \\
\grave{1} \\
\vdots \\
0\end{array}$ & ما & - & ما & L & 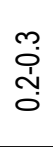 \\
\hline & 意 & $\overline{\tilde{U}}$ & ฮี & 艺 & 롱 & $\left|\begin{array}{l}0 \\
0 \\
0\end{array}\right|$ & 气ิ & & $\frac{m}{\alpha}$ & 몽 & $\stackrel{\text { م }}{\alpha}$ \\
\hline & 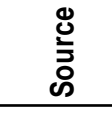 & \multicolumn{6}{|c|}{ iе sпіпм/олиоэ } & \multicolumn{4}{|c|}{ un!qә|d unuo6א/od } \\
\hline & 莘 & - & $\sim$ & $m$ & $\nabla$ & م & 0 & $r$ & $\infty$ & $\sigma$ & 으 \\
\hline
\end{tabular}




\section{Plant-Microbe Interactions}

To determine beneficial interactions between plants and microbes, PMI assay was done. Zea mays seeds were inoculated with the bacterial strains ( $\mathrm{Cn} 1$, Bacillus subtilis (Cn2), Cn4, Cn5, Streptomyces lydicus (Cn6), Staphylococcus aureus (Cn7), PP2, Bacillus pumilus (PP3), PP4 and PP5) and growth parameters of plants e.g., fresh weight, shoot length and root length were noted to estimate the growth promotional potential of these strains. Considerable increase in fresh weight of plants was observed in strains PP2, Cn1, Cn5, Cn4 and Streptomyces lydicus (Cn6) showing 94.36, 74.50, 74.21, 70.41 , and $65.03 \%$ increase respectively as compared to control (Figure 4). Treatment with strains namely Cn1, $\mathrm{Cn} 5$, Bacillus subtilis (Cn2) and PP2 showed significant percentage increase in shoot length of up to 26.12, 23.23, 21.37 and $21.0 \%$ respectively over control. But the two strains Bacillus pumilus (PP3) and PP5 showed percentage decrease in shoot length over control (Figure 5). A careful study demonstrated that some bacterial strains namely $\mathrm{Cn} 5$, Streptomyces lydicus ( $\mathrm{Cn} 6)$, Bacillus subtilis (Cn2), Cn4 and PP4 showed significant percentage increase in root length of upto $64.95,64.60$, $53.22,50.74$ and $50.39 \%$ respectively, compared to control (Figure 5).

Analysis of chlorophyll 'a', 'b' and total chlorophyll content was done. Few strains namely $\mathrm{Cn} 5, \mathrm{Cn} 4$, Streptomyces lydicus (Cn6) and PP2 showed significant percentage increase in chlorophyll 'a' of upto $100,99.9,82.80$ and $80.99 \%$ respectively, then non-inoculated control plants. Four strains namely $\mathrm{Cn} 5, \mathrm{Cn} 4, \quad$ Streptomyces lydicus ( $\mathrm{Cn} 6$ ) and PP2 showed maximum chlorophyll 'b' concentration in treated plants with percentage increase of $100,99.72,82.84$ and $80.95 \%$ respectively over control. Bacterial strains namely $\mathrm{Cn} 5, \mathrm{Cn} 4$, Streptomyces lydicus ( $\mathrm{Cn} 6$ ) and PP2 showed maximum total chlorophyll content in treated plants with a percentage increase of $100,99.82,82.81$ and $80.96 \%$ respectively over control. Exceptionally, Bacillus subtilis ( $\mathrm{Cn} 2$ ) showed percentage decrease in chlorophyll (a, b and total chlorophyll) concentration over control (Figure 6).

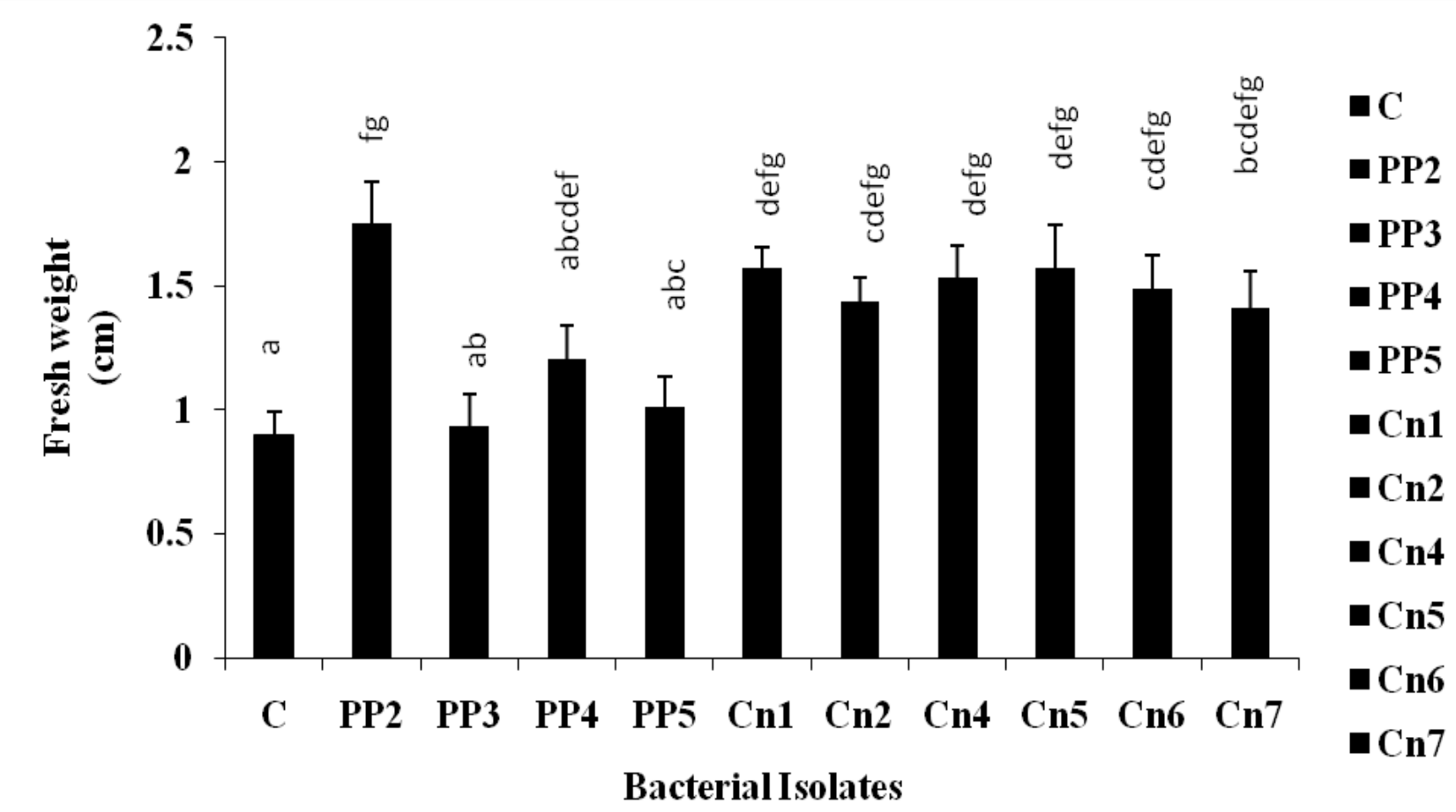

Figure 4. Effect of bacterial inoculations on the fresh weight of Zea mays under laboratory conditions. Data represent mean of twelve replicates. Different letters indicate significant differences between treatments using Duncan's multiple range test $(\mathrm{P}=0.05)(\mathrm{C}=$ Control). 


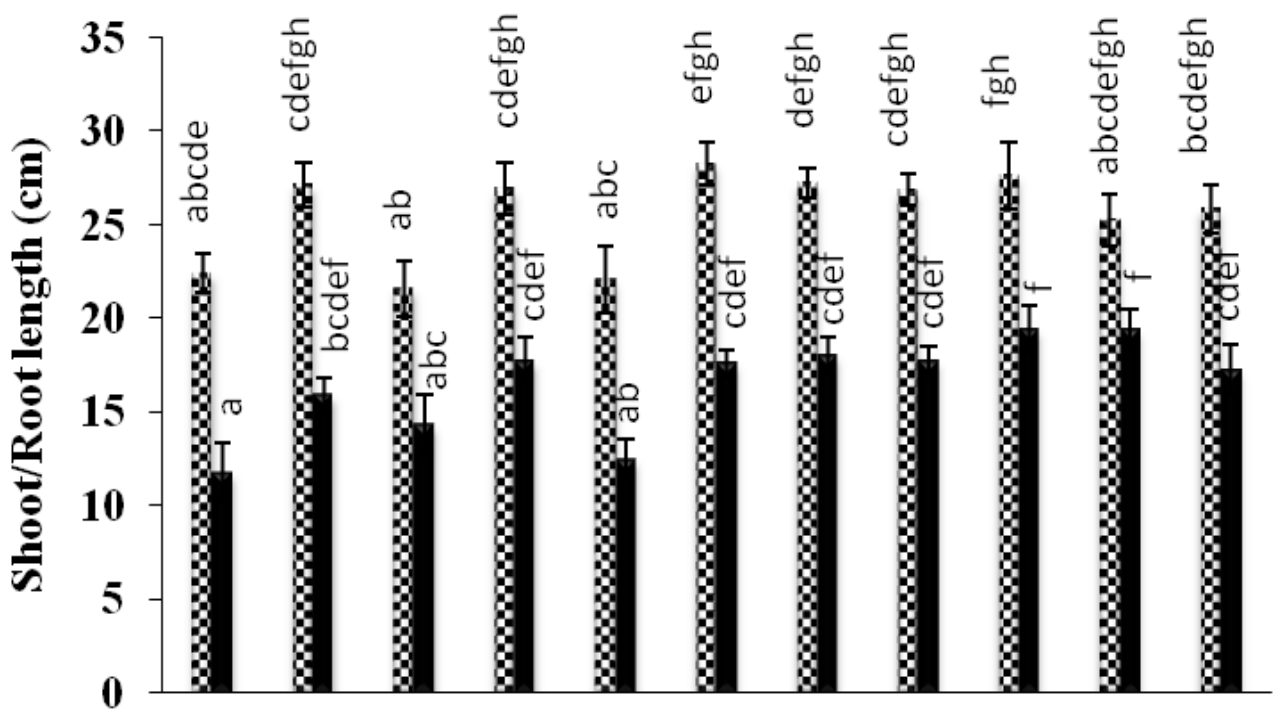 \\ C $\quad$ PP2 PP3 PP4 PP5 Cn1 Cn2 Cn4 Cn5 Cn6 Cn7 \\ Bacterial isolates}

\& Shoot length

Root length

Figure 5. Effect of bacterial inoculations on shoot/root length of Zea mays under laboratory conditions. Data represent mean of twelve replicates. Different letters indicate significant differences between treatments using Duncan's multiple range test $(P=0.05)(C=$ Control).

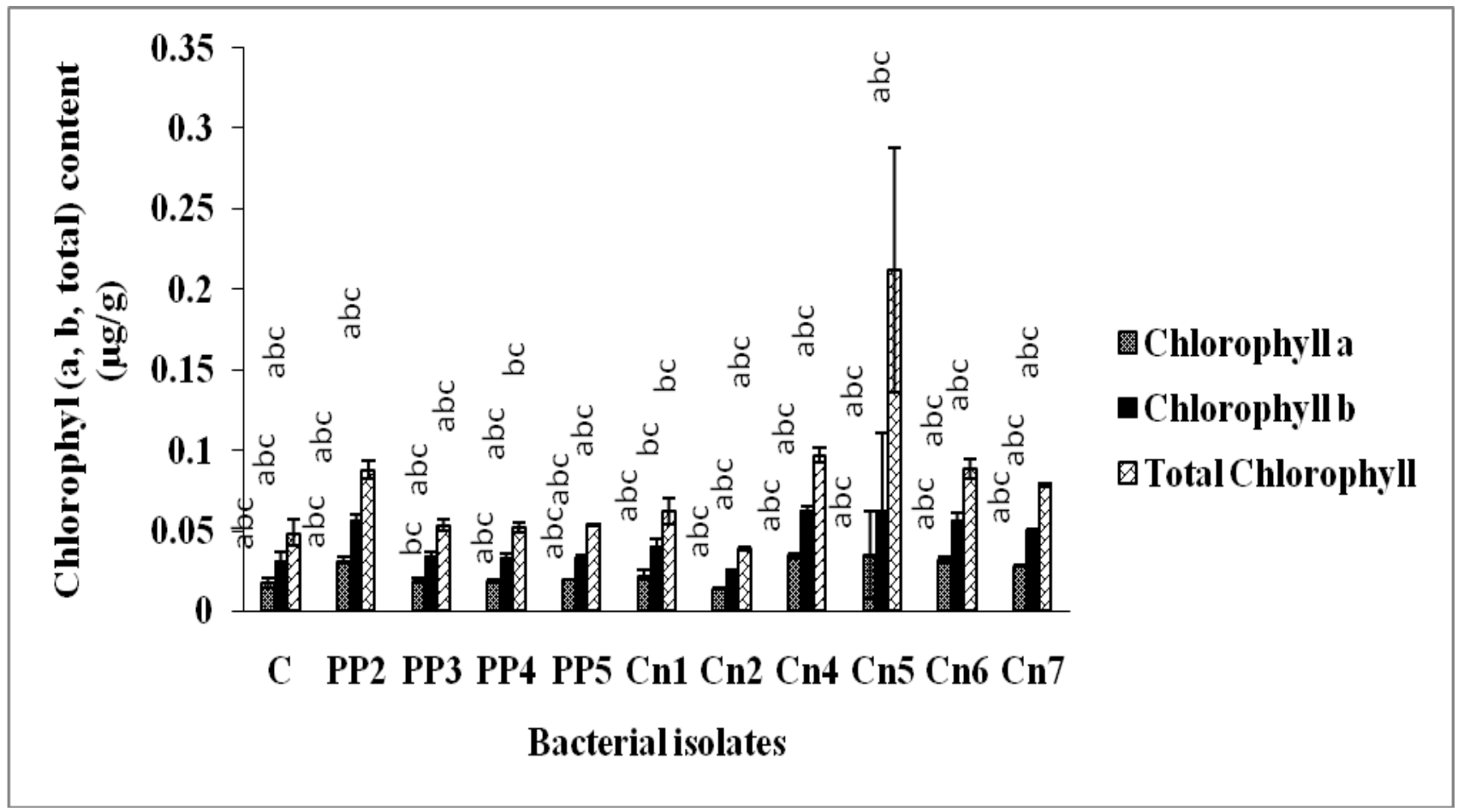

Figure 6. Effect of bacterial inoculations on chlorophyll 'a' of Zea mays under laboratory conditions. Data represent the mean of three replicates. Different letters indicate significant differences between treatments using Duncan's multiple range test $(\mathrm{P}=0.05)(\mathrm{C}=$ Control). 


\section{DISCUSSION}

The current study focused on determining the plant growth-promoting potential of rhizobacteria isolated from the rhizosphere of Convolvulus arvensis and Polygonum plebeium collected from mountainous region of the Zhob district of Balochistan including four already isolated and identified strains. As Balochistan is not an agricultural land so our purpose was to develop methods using rhizobacteria from Balochistan native plants to enhance the productivity of that region.

In PMI assay, fresh weight, root length and shoot length of inoculated and control plants were observed. A maximum increase in fresh weight of plants was observed in strains PP2 (94.36\%), which was observed to be a strong HCN producer and ammonia producer, both of which directly and indirectly contributed to an increase in growth parameters. In addition, all the treated plants showed percentage increase in fresh weight relative to control plant (Figure 4). This was due to individual or synergistic effects of plant growth-promoting traits of rhizobacteria, which modified the uptake of minerals by plants $^{8}$. Similarly the inoculation of Zea mays seeds with rhizobacterial strains showed a noteworthy increase in shoot length and root length. $\mathrm{Cn} 1, \mathrm{Cn} 5$ and few other strains showed a significant percentage increase in shoot length. PGPR may help plant growth promotion by using their hormones producing ability, nitrogen fixation or even phosphate solubilization. They may also affect plant metabolism to increase water and minerals uptake, by suppressing plant pathogens i.e. through $\mathrm{HCN}$ production or even by collaborating with other beneficial microorganisms ${ }^{4}$. Balseiro-Romero et al. (2017) and many other researchers working on the effect of PGPR on growth parameters of various plants reported that shoot length increased in their experiments ${ }^{20}$. The present study demonstrated that out of the ten bacterial strains, five bacterial strains namely $\mathrm{Cn} 5$, Streptomyces lydicus (Cn6), Bacillus subtilis (Cn2), $\mathrm{Cn} 4$ and PP4 showed considerable percentage increase of more than $50 \%$ in root length over control. Cn5 and PP4 neither produced ammonia nor auxin but these were $\mathrm{HCN}$ producers and showed considerable increase in root length because $\mathrm{HCN}$ provides indirect mechanism of protection by controlling other pathogens. Moreover, the presence of other plant growth-promoting traits may be one of the causes, as these may also be siderophore producers and phosphate solubilizers $^{21}$ (Figure 5). Zahid et al. (2015) reported similar results of increase in root length in Zea mays after bacterial treatment 22 .

All the bacterial strains showed increased chlorophyll content in inoculated plants than control plants except for Bacillus subtilis ( $\mathrm{Cn} 2)$. Actually, the bacterial inoculated plants which showed a significant increase in fresh weight, and shoot/root length were also rich in chlorophyll content over control. PGPR inoculated plants showed dark greenish leaves, which were due to an increase in chlorophyll content ${ }^{23}$. It can be assumed that the increase in chlorophyll content was due to increased leaf area and balanced nutritional uptake from the soil24. Uzair et al. (2018) worked on 18 different strains of $P$. aeruginosa isolated from Balochistan and tested these for their plant growth-promoting potential and obtained significant results ${ }^{25}$. Sarwar et al. (2014) also worked on PGPR isolated from rhizosphere of rice plants of Jafferabad district of Balochistan and revealed significant increase in plant parameters ${ }^{26}$. Our results are in accordance with the findings of Kamran et al. (2016) who worked on PGPR strain i.e., Pseudomonas putida and reported significant increase in chlorophyll content of Eruca sativa. They revealed that plant growth-promoting bacteria supported the defense mechanism to produce various enzymes that ultimately increase the chlorophyll content of treated plants $^{27,28}$.

\section{CONCLUSION}

From the so forth experimental work, it is deduced that PGPR are beneficial for the growth promotion of plants. Our results revealed that bacteria isolated from plant samples, collected from unproductive areas of Baluchistan were ammonia producing and possessed the ability to produce $\mathrm{HCN}$, an important plant growth promoter, while only two bacteria were auxin producers. These bacteria were then treated with Zea mays L., which showed significant enhancement in plant growth parameters including chlorophyll content of plants exhibiting beneficial behavior of bacteria towards plant traits. Hence, the present study gives an idea to enhance plant productivity on such barren lands that can be utilized for agricultural purposes. 


\section{ACKNOWLEDGEMENTS}

This work was funded by the grant from the University of the Punjab, Quaid-e-Azam Campus, Lahore.

\section{LIST OF ABBREVIATIONS}

PGPR

Plant Growth Promoting Rhizobacteria

$\begin{array}{ll}\mathrm{N}_{2} \mathrm{O} & \text { Nitrous Oxide } \\ \mathrm{L}-\text { Agar } & \text { Luria Agar } \\ \text { L- Broth } & \text { Luria Broth }\end{array}$

\section{REFERENCES}

1. Bulgarelli D, Schlaeppi K, Spaepen S, Van Themaat $E V$, Schulze-Lefert P. Structure and functions of the bacterial microbiota of plants. Annu Rev Plant Biol. 2013; 64:807-38.

2. Haldar $\mathrm{S}$, Sengupta S. Plant-microbe cross-talk in the rhizosphere: insight and biotechnological potential. Open Microbiol J. 2015; 9:(1):1-7.

3. Hardoim PR, Van Overbeek LS, Berg G, Pirttilä AM, Compant S, Campisano A, et al. The hidden world within plants: ecological and evolutionary considerations for defining functioning of microbial endophytes. Microbiol Mol Biol Rev. 2015;79(3):293320.

4. Pérez-Montaño $F$, Alías-Villegas $C$, Bellogín RA, Del Cerro P, Espuny MR, Jiménez-Guerrero I, et al. Plant growth promotion in cereal and leguminous agricultural important plants: from microorganism capacities to crop production. Microbiol Res. 2014; 169(5-6):325-36.

5. Nadeem SM, Ahmad M, Zahir ZA, Javaid A, Ashraf $M$. The role of mycorrhizae and plant growth promoting rhizobacteria (PGPR) in improving crop productivity under stressful environments. Biotechnol Adv.2014; 32(2):429-48.

6. Chamam A, Sanguin H, Bellvert F, Meiffren G, Comte $G$, Wisniewski-Dyé $F$, et al. Plant secondary metabolite profiling evidences strain-dependent effect in the Azospirillum-Oryza sativa association. Phytochem. 2013; 87:65-77.

7. Kechid M, Desbrosses G, Rokhsi W, Varoquaux F, Djekoun A, Touraine B. The NRT 2.5 and NRT 2.6 genes are involved in growth promotion of Arabidopsis by the plant growth-promoting rhizobacterium (PGPR) strain Phyllobacterium brassicacearum STM 196. New Phytol. 2013; 198(2):514-24.

8. Pérez-de-Luque A, Tille S, Johnson I, Pascual-Pardo $D$, Ton J, Cameron DD. The interactive effects of arbuscular mycorrhiza and plant growth-promoting rhizobacteria synergistically enhance host plant defences against pathogens. Sci Rep.2017; 7(1):16409-19.

9. Vacheron J, Desbrosses G, Bouffaud ML, Touraine B, Moënne-Loccoz Y, Muller D, et al. Plant growthpromoting rhizobacteria and root system functioning. Front Plant Sci. 2013; 4:356.

10. Dal Cortivo C, Barion G, Visioli G, Mattarozzi M, Mosca G, Vamerali T. Increased root growth and nitrogen accumulation in common wheat following PGPR inoculation: Assessment of plant-microbe interactions by ESEM. Agric Ecosyst Envirn. 2017; 247:396-408.

11. Myresiotis CK, Vryzas Z, Papadopoulou-Mourkidou E. Biodegradation of soil-applied pesticides by selected strains of plant growth-promoting rhizobacteria (PGPR) and their effects on bacterial growth. Biodegradation. 2012; 23(2):297-310.

12. Bhattarai $H R$, Virkajärvi $P$, YliPirilä $P$, Maljanen $M$. Emissions of nitrous acid (HONO), nitric oxide (NO) and nitrous oxide $\left(\mathrm{N}_{2} \mathrm{O}\right)$ from boreal agricultural soilEffect of $\mathrm{N}$ fertilization. In: EGU General Assembly Conference Abstracts. 2017. Vol. 19, 3221.

13. Gupta G, Parihar SS, Ahirwar NK, Snehi SK, Singh V. Plant growth promoting rhizobacteria (PGPR): current and future prospects for development of sustainable agriculture. J Microb Biochem Technol. 2015; 7(2): 96-102.

14. Vafadar F, Amooaghaie R, Otroshy M. Effects of plant-growth-promoting rhizobacteria and arbuscular mycorrhizal fungus on plant growth, stevioside, NPK, and chlorophyll content of Stevia rebaudiana. J Plant Interact. 2014; 9(1):128-36.

15. Myresiotis CK, Vryzas Z, Papadopoulou-Mourkidou E. Effect of specific plant-growth-promoting rhizobacteria (PGPR) on growth and uptake of neonicotinoid insecticide thiamethoxam in corn (Zea mays L.) seedlings. Pest Manag Sci. 2015; 71(9):1258-66.

16. Fatima $\mathrm{H}$, Ahmed $A$. How chromium-resistant bacteria can improve corn growth in chromium contaminated growing medium. Pol J Environ Stud. 2016; 25(6): 2357-65.

17. Microbiology: A Laboratory Manual (third ed), Benjamin/cummings Pub. Co. New York (1992), 125-79.

18. Lorck $H$. Production of hydrocyanic acid by bacteria. Physiologia Plantarum. 1948; 1(2):142-46.

19. Ahmed A, Hasnain S. Auxin-producing Bacillus sp.: Auxin quantification and effect on the growth of Solanum tuberosum. Pure Appl Chem. 2010; 82(1):313-9.

20. Balseiro-Romero M, Gkorezis P, Kidd PS, Van Hamme J, Weyens N, Monterroso C, et al. Use of plant growth promoting bacterial strains to improve Cytisus striatus and Lupinus luteus development for potential application in phytoremediation. Sci Tot Envir. 2017; 581:676-88. 
21. Souza RD, Ambrosini A, Passaglia LM. Plant growthpromoting bacteria as inoculants in agricultural soils. Genet Mol Biol. 2015; 38(4): 401-19.

22. Zahid M, Abbasi MK, Hameed S, Rahim N. Isolation and identification of indigenous plant growth promoting rhizobacteria from Himalayan region of Kashmir and their effect on improving growth and nutrient contents of maize (Zea mays L.). Front Microbiol. 2015; 6: 207-17.

23. Habib SH, Kausar H, Saud HM. Plant growthpromoting rhizobacteria enhance salinity stress tolerance in okra through ROS-scavenging enzymes. Biomed Res Int. 2016; 2016: 6284547.

24. Seema K, Mehta K, Singh N. Studies on the effect of plant growth promoting rhizobacteria (PGPR) on growth, physiological parameters, yield and fruit quality of strawberry cv. Chandler. 2018; 7(2): 383-7.

25. Uzair B, Kausar R, Bano SA, Fatima S, Badshah M, Habiba $U$, et al. Isolation and Molecular
Characterization of a Model Antagonistic Pseudomonas aeruginosa Divulging In Vitro Plant Growth Promoting Characteristics. Bio Med Res Int. 2018; 2018: 6147380-7.

26. Sarwar A, Sajid I, Riaz R, urRehman F, Ahmed SS, Shahwani N, et al. Bacterial community profiles in rhizosphere of paddy rice based on 16s rRNA sequence analysis. Int J Biol Biotech. 2014; 12(1):119.

27. Kamran MA, Bibi S, Xu RK, Hussain S, Mehmood K, Chaudhary HJ. Phyto-extraction of chromium and influence of plant growth promoting bacteria to enhance plant growth. J Geochem Explor. 2017; 182: 269-74.

28. Habib S, Fatima H, Ahmed A. Comparative Analysis of Pre-Germination and Post-Germination Inoculation Treatments of Zea mays L. to Mitigate Chromium Toxicity in Cr-Contaminated Soils. Pol J Environ Stud. 2019; 28(2): 597-607. 